\title{
Liposomal amphotericin B in hepatic candidosis
}

\author{
M Sharland, R J Hay, E G Davies
}

\begin{abstract}
A 4 year old girl treated with a standard chemotherapy protocol for acute lymphoblastic leukaemia developed hepatic candidosis during the consolidation phase. This relapsed after a prolonged course of amphotericin $B$ and flucytosine. An eight week course of liposomal amphotericin produced a marked clinical improvement which was sustained for one year. A subsequent relapse was associated with transformation to myelodysplastic leukaemia.
\end{abstract}

(Arch Dis Child 1994; 70: 546-547)

In children with leukaemia, aggressive chemotherapy with prolonged neutropenia is associated with an increased incidence of systemic fungal infections. ${ }^{1}$ A syndrome of hepatic candidosis due to fungal microabscesses has been increasingly reported in neutropenic children. ${ }^{2}$ The clinical diagnosis is suggested by a persistent fever, right upper quadrant abdominal pain, increased plasma alkaline phosphatase, and hepatosplenomegaly. Blood cultures are usually negative. A characteristic appearance may be seen on ultrasound or computed tomography. The diagnosis can be made by a needle ${ }^{1}$ or open wedge liver biopsy sample, with yeasts and pseudohyphae seen and cultured. ${ }^{3}$ The standard treatment is amphotericin B with flucytosine. A high rate of treatment failure and side effects are seen in hepatic candidosis. A novel preparation in which amphotericin B is covalently bound into unilamellar liposomes (AmBisome) offers the possibility of reduced toxicity and increased efficacy. ${ }^{4}$

\section{Case report}

A 4 year old Saudi Arabian girl presented with lymphadenopathy and hepatosplenomegaly. A bone marrow biopsy sample confirmed common acute lymphoblastic leukaemia. She became pyrexial after one week of induction chemotherapy, with no response to antibiotic treatment. One month later she remained febrile and had become jaundiced with increased hepatosplenomegaly; all chemotherapy was stopped because of deteriorating liver function. An empirical two week course of gancyclovir produced no benefit and the patient was transferred to the UK.

On arrival at St George's Hospital the child was noted to be deeply jaundiced, with massive hepatomegaly, a moderately enlarged spleen, and oral candidosis. Abdominal ultrasound showed multiple brightly reflective foci. Bone marrow aspiration was normal and an open wedge liver biopsy sample showed the formation of suppurative granuloma, with yeasts and pseudomycelium, confirming the diagnosis of hepatic candidosis. No growth was obtained either from the liver or multiple blood cultures. The child was treated with amphotericin B to a total dose of $0.75 \mathrm{~g}$ and flucytosine. This treatment produced severe systemic reactions and hypokalaemia. The fever and jaundice resolved, and the hepatosplenomegaly improved with treatment. Further treatment for leukaemia was then begun, and the child returned to Saudi Arabia while receiving fluconazole by mouth.

Three months after her return home, her fever returned, with progression of hepatomegaly, despite continuing oral fluconazole. She therefore returned to the UK, where a repeat open liver biopsy sample showed active candida infiltration. A six week course of itraconazole and flucytosine had no effect on her fever or clinical status. A range of immune function tests were normal.

Liposomal amphotericin (AmBisome) was given daily for two months to a total dose of $5 \mathrm{~g}$, producing no systemic reaction and only mild hypokalaemia. Over the treatment period the child's fever settled, she gained weight, and her hepatomegaly improved. Oral fluconazole was again started and the patient remained well for one year.

The child presented again with anaemia and thrombocytopenia. Although she was not jaundiced a bone marrow sample showed myelodysplasia. A further six week course of AmBisome was given, followed by splenectomy and an open liver biopsy sample. Active areas of candidal infection were seen in the liver. Unfortunately, over the next month blast cells were detected in her blood due to myelomonocytic leukaemia. The child therefore returned home for palliative care, and died eight months later.

\section{Discussion}

In hepatic candidosis treatment failure is a major problem. In adults with hepatic candidosis optimally treated with amphotericin $B$, approximately one third of patients die with active fungal disease. ${ }^{3}$ The failure of this child to respond to high doses of amphotericin B was probably due to the difficulty in achieving effective hepatic levels.

In an attempt to improve tissue delivery and reduce systemic toxicity, amphotericin has been incorporated into liposomes. These are closed spherical vesicles made of sterols and phospholipids. With the formulation used in our patient (AmBisome), the liposomes are rapidly cleared from the blood and taken up by the reticuloendothelial system. High concentrations are therefore achieved in
St Thomas's Hospita

London

Correspondence to: Dr Sharland.

Accepted 2 March 1994 
organs rich in reticuloendothelial cells, including the liver and spleen, while the concentration in the kidneys is low. ${ }^{4}$ The rapid dispersion and 'targeting' of the amphotericin has the potential to reduce toxicity (especially renal) and increase the maximum possible total dose.

There have been few clinical trials of liposomal amphotericin. A large, multicentre, phase II/III trial of AmBisome evaluated over 100 immunocompromised patients with invasive fungal infection. ${ }^{5}$ All patients had previously received and then stopped treatment with amphotericin $\mathrm{B}$ because of toxicity or treatment failure. Liposomal amphotericin produced an overall cure in $58 \%$ of patients, with an eradication rate of $83 \%$ for candida species. The dose of liposomal amphotericin in treating hepatic candidosis in childhood is unclear. Doses of up to $6 \mathrm{mg} / \mathrm{kg} /$ day have been tolerated. ${ }^{6} \mathrm{~A}$ prolonged course of treatment is necessary, which should be followed by maintenance treatment with fluconazole.

There are now several lipid suspensions of amphotericin under evaluation which offer a novel therapeutic approach to invasive fungal disease. They are expensive, and direct comparison clinical trials are required to determine whether they should replace conventional amphotericin as the initial treatment of all fungal sepsis. In hepatic candidosis the poor results with conventional treatment argue strongly in favour of using liposomal amphotericin $\mathbf{B}$ as a first line treatment.

We thank Professor A Mowat and Professor J Chessells for helpful advice in the clinical management of this patient.

1 Pizzo PA, Walsh TJ. Fungal infections in the paediatric cancer patient. Semin Oncol 1990; 17 (suppl 6): 6-9.

2 Verdeguer A, Fernandez JM, Esquembre C, Ferris J, Ruiz JG, Castel V. Hepatosplenic candidiasis in children with acute leukaemia. Cancer 1990; 65: 874-7.

3 Haron E, Feld R, Tuffnell P, Patterson B, Hasselback R, Matlow A. Hepatic candidiasis: an increasing problem in immunocompromised patients. $A m \mathcal{F}$ Med 1987; 83: 17-26.

4 Gregoriadis G. Overview of liposomes. $f$ Antimicrob Chemother 1991; 28 (suppl B): 39-48.

5 Ringden O, Meunier F, Tollemar J, et al. Efficacy of amphotericin B encapsulated in liposomes (AmBisome) in the treatment of invasive fungal infection in immunocompromised patients. F Antimicrob Chemother 1991; 28 (suppl B): 73-82.

6 Zoubek A, Emminger W, Emminger-Schmidmeier W, et al. Conventional vs liposomal amphotericin B in immunosuppressed children. Pediatr Hematol Oncol 1992; 9: suppressed $187-93$. 